\title{
Atomic-Resolution Imaging and In-situ EELS Study of Ferroelastic and Ferromagnetic Ordering in $\mathrm{LaCoO}_{3}$
}

\author{
T. Yuan ${ }^{1}$, N. Orlovskaya ${ }^{2}$, and R. F. Klie ${ }^{1}$ \\ ${ }^{1}$ Department of Physics, University of Illinois at Chicago, Chicago, IL, 60607 \\ ${ }^{2}$ Department of Mechanical, Material, and Aerospace Engineering, University of Central \\ Florida, Orlando, FL, 32816
}

The perovskite oxide $\mathrm{LaCoO}_{3}$ has attracted increasing attention due to its reported roomtemperature ferroelastic behavior, ${ }^{1}$ and a ferromagnetic transition observed at around $80 \mathrm{~K}$ in epitaxially strained thin films. ${ }^{2}$ To advance our understanding of these properties, a combination of atomic-resolution Z-contrast imaging and electron energy-loss spectroscopy (EELS) with insitu cooling experiments has been used to study the $\mathrm{LaCoO}_{3}$ microstructures associated with the multiple ferroic transitions in bulk $\mathrm{LaCoO}_{3}$.

In particular, $\mathrm{LaCoO}_{3}$ exhibits a hysteretic stress-strain behavior as a ferroelastic material at room temperature. The coercive stress marks the point where the ferroelastic transitions occur. Therefore, we study polycrystalline $\mathrm{LaCoO}_{3}$ samples compressed both above and below the coercive stress. By energy-loss magnetic circular dichroism (EMCD) method, we obtained angular-resolved EELS of Co L-edges at low temperature in the diffraction mode for $\mathrm{LaCoO}_{3}$ compressed below and above the coercive stress. ${ }^{3}$ (Figure 1) It suggest that there may be ferromagnetic transitions at low temperature for the polycrystalline $\mathrm{LaCoO}_{3}$ sample compressed above the coercive stress.

Moreover, for untreated and compressed polycrystalline bulk $\mathrm{LaCoO}_{3}$ samples, we find that both $\mathrm{Co}_{3} / \mathrm{L}_{2}$ ratios are slightly different at room temperature and low temperature. (Figure 2) On the other hand, the Co L-edges does not change at room temperature and low temperature in pure single crystalline $\mathrm{LaCoO}_{3}$. This unusual result may be caused by defects, oxygen vacancies or large concentration of twinning structure found in polycrystalline bulk $\mathrm{LaCoO}_{3}$. (Figure 3) $\mathrm{We}$ study these defects in the polycrystalline $\mathrm{LaCoO} 3$ by atomic-resolution EELS. ${ }^{3}$

In this presentation, we will show how the microstructures in $\mathrm{LaC}_{-} \mathrm{O}_{3}$ influence the ferromagnetic order in $\mathrm{LaCoO}_{3}$. We will also explore the relationship between the observed ferroelastic and ferromagnetic properties of $\mathrm{LaCoO}_{3}{ }^{4}$

References:

1. M. Lugovy, V. Slyunyayev, N. Orlovskaya, D. Verbylo, and M. Reece, Phys. Rev. B 78, 024107 (2008)

2. D. Fuchs, C. Pinta, T. Schwarz, P. Schweiss, P. Nagel, S. Schuppler, R. Schneider, M. Merz, G. Roth, and H. Lohneysen, Phys. Rev. B 75, 144402 (2007) 
"In situ EELS study of the ferromagnetic properties in ferroelastical polycrystalline bulk $\mathrm{LaCoO}_{3}$ ", T. Yuan, R. F. Klie, To be submitted

${ }^{4}$ Funded by: NSF CAREER Award DMR-0846748
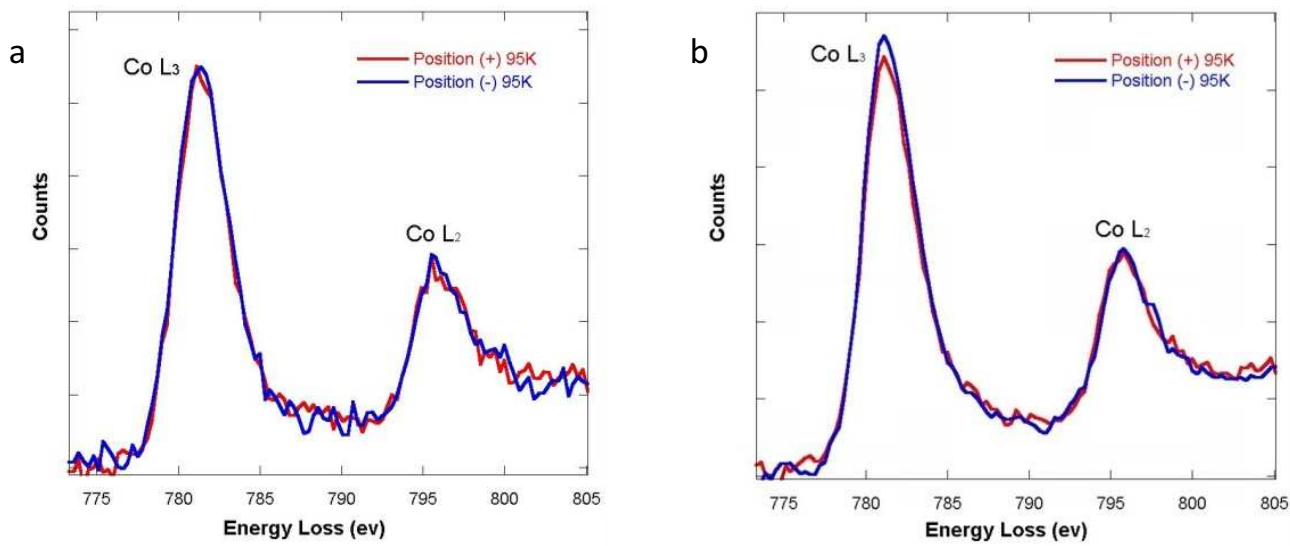

Figure 1: Angular-resolved EELS of Co L-edges at low temperature in $\mathrm{LaCoO}_{3}$ compressed (a) below (b)above coercive stress following EMCD method
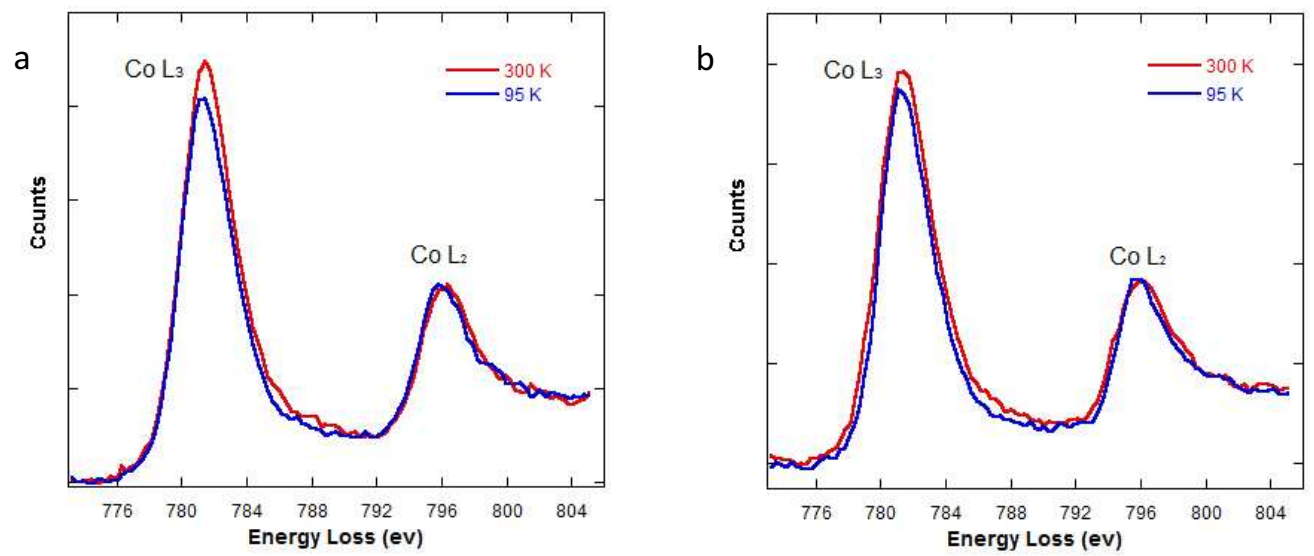

Figure 2: Angular-resolved EELS of Co L-edges for (a) untreated (b) compressed polycrystalline $\mathrm{LaCoO}_{3}$ sample at room temperature and low temperature

a

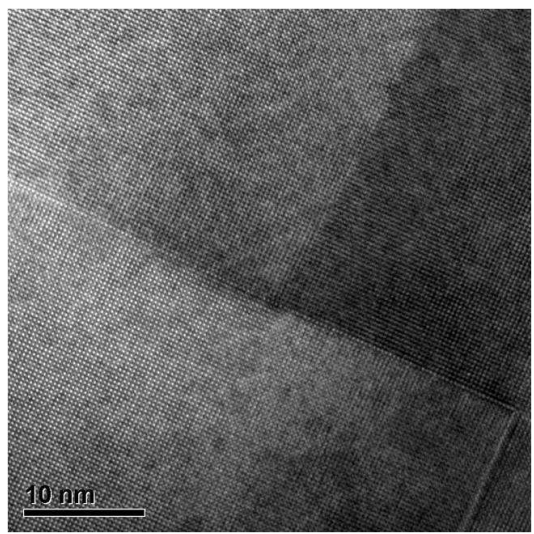

b

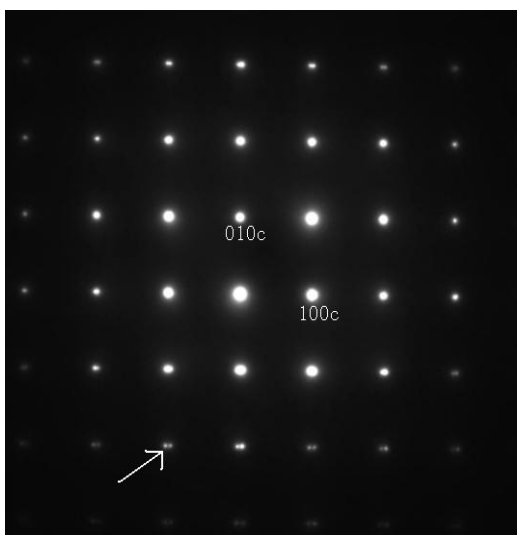

Figure 3: (a) High resolution image and (b) diffraction pattern of the twinning structure along $\left[\begin{array}{lll}1 & 0 & 0\end{array}\right]$ direction in untreated polycrystalline $\mathrm{LaCoO}_{3}$ bulk sample. The splitting peak pointed by an arrow show the existence of the twinning structure. 